\title{
Bioengineering Of Antibodies Marked With Atoms Of Different Elements For Simultaneous Localization Of Biomolecules With Electron Energy Loss Spectroscopic Imaging
}

\author{
Marek Malecki, Marion Greaser, and Ralph Albrecht \\ University of Wisconsin, Madison, WI 53705, USA
}

Identification of components in macromolecular assemblies is an essential step toward the determination of their interactions and functions. The goal for such an effort is to create a bridge between biochemistry and molecular biology data with images of molecular architecture showing distribution and co-localization of specific molecular species. Bioengineered antibodies carrying reporter elements either as atoms individually incorporated into or as small colloidal particles attached to these antibodies can be used to attain such a goal. We have previously pioneered development of labels, which permit, by means of spectroscopy, simultaneous detection and localization of several different molecules within one frame [1-3]. In order to confirm the capabilities of the system, we have employed a model using a molecule of alpha-actinin. It serves as anchoring site for actin and has a precisely known periodic pattern of distribution within myofibril architecture. Antibodies against alpha-actinin were derivatized with boron [1,2]. Rabbit psoas muscle was prepared via high-pressure freezing, followed by freeze-substitution, epon-embedding, and sectioning [4]. Alpha-actinin in sections was labeled with boronated antibodies [2]. The samples were viewed with the energy filtering transmission electron microscope (LEO 912 EFTEM) equipped with the omega energy filter and EELS calculation software (SIS 2.2). Distribution of boronated antibodies against alpha-actinin was determined based upon EELS imaging maps for boron at $201 \mathrm{eV}$ edge. The pattern of alpha-actinin could be clearly demonstrated (Fig. 1). That pattern was compared to the overall ultrastructure revealed through the contrast tuning near the carbon edge (Fig.2). The label distribution can be superimposed onto myofibril architecture and the distribution of alpha-actinin within myofibrillar architecture concluded. The distribution of boronated anti-alpha-actinin antibodies is restricted to the Z-line validating the known to be a pattern of alpha-actinin localization. This demonstrates both the accuracy and fidelity attainable using labeled bioengineered antibodies in conjunction with EELS imaging. [5]

\section{References.}

[1] M. Malecki et al. Proc. Nat. Acad. Sci. USA 99 (2002) 213.

[2] M. Malecki M. Sci. of Spec. Prep. for Micros.\& Microa. 10 (1996) 1.

[3] D.A. Meyer and R.M. Albrecht, Micros. \& Microa. 6 (Suppl. 2) (2000) 322.

[4] M. Malecki \& M. Greaser Scan. Micr. 7, 1 (1992) 115.

[5] Supported in part by NIH/NIGMS \#63001.

\section{Figure legends.}

Fig. 1. Distribution of boronated antibodies against alpha-actinin, which was determined based upon EELS imaging maps for boron at $201 \mathrm{eV}$ edge in the LEO 912 Omega EFTEM. Areas of Z-line illustrated on top of Fig. 2 were selected for mapping. Notice very dense and specific labeling with anti-alpha-actinin antibodies, lack of unspecific labeling, clean background, and strong signal-tonoise ratio. Fig. 2. Overall ultrastructure of the labeled muscle. This image was obtained through the contrast tuning near the carbon edge and reversing contrast. Horizontal field width $775 \mathrm{~nm}$. 


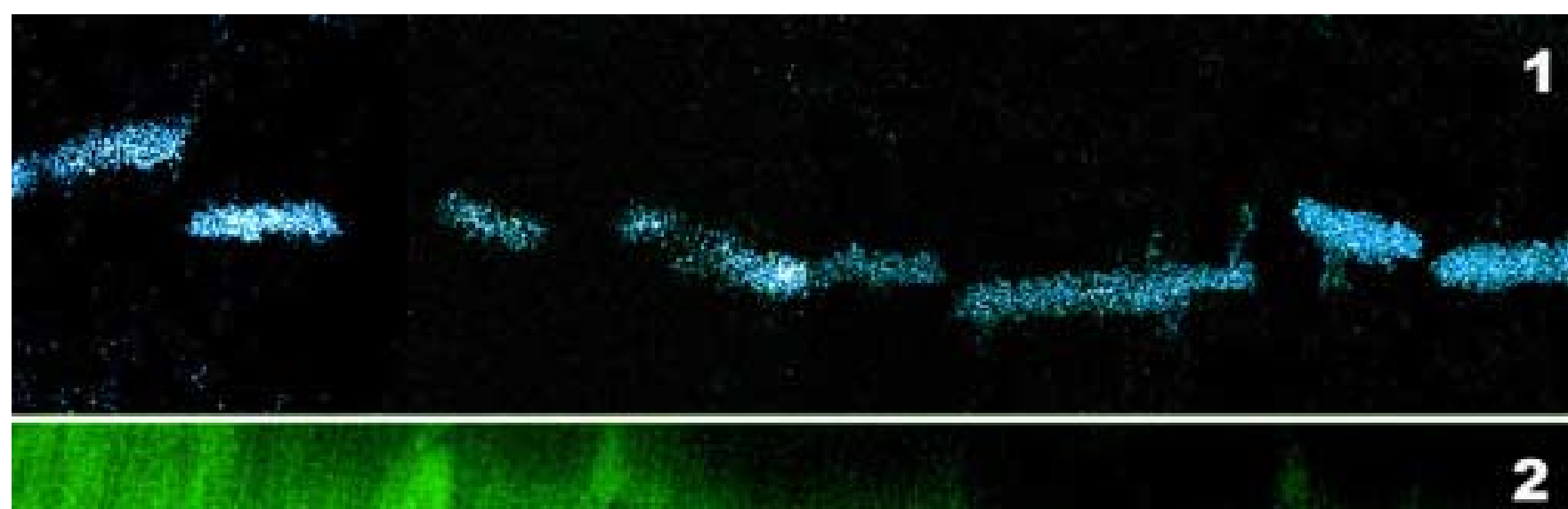


https://doi.org/10.1017/S1431927603445960 Published online by Cambridge University Press 